\title{
An Analysis of Lexical Features in Advertising English from the Mental Lexicon Theory \\ Qiaoli Li
}

Department of Basic Sciences of Shaanxi Institute of International Trade >\& Commerce. 712046

Keywords: English; Advertising language; Mental lexicon

\begin{abstract}
The vocabulary is the basic unit of the advertising language. As a one-way communication which is community-oriented, advertising requires appropriate representation of the language in order to achieve the expected effect of publicity. The construction of English advertising language is based on psycholinguistics. The theory of mental lexicon has found some features of vocabulary acquisition. This paper intends to analyze the possible application of the theory in advertising English and factors influencing the word extraction to advertising designer and readers.
\end{abstract}

\section{Introduction}

With the development of global economy, countries around the world are all throwing themselves into the international business. As an effective vehicle of promoting sales, providing services and building images, advertising plays an essential role in modern life.

Advertising is not only a way to persuade customers to buy goods so as to explore market and make profits, but also a cultural meaning communication process. Advertising language generally contains specific meaning of a certain culture and cultural connotations. Advertising maintains a significant achievement of country's history, values, customs, national character, etc. It gives us a full view of whatever the culture has created and whatever the concepts and ideas the culture advocates. Advertising transforms cultural values, beliefs, attitudes, and actions.

Choice of words in advertising is the key factor to a good or successful advertisement. Psycholinguists examine the relationship between words and meaning from another angle: how people save ewords in memory; how to identify and extract the words, how to understand the meaning of the words. These processes all have relation with psychology, which is called mental lexicon. This paper will study the word extraction of advertising English on basis of mental lexicon theory.

\section{Literature Review}

Definition and Classification of Advertisement. Advertisement is not only an economic activity, but also a cultural communication. Then what is advertisement? There are various definitions of advertisement from different perspectives or according to different purposes. Albert Lasker, the father of modern advertisement, said advertisement was "salesmanship in print." His words suggest that the ultimate objective of advertisement is to sell. However, with the development of media and the expansion of the nature and scope of advertisement, his saying is quite limited.

Encyclopedia Britannica defines advertisement as follows: "Advertisement is a form of communication intended to promote the sale of a product or service, to influence public opinion, to gain political support, to advance a particular cause, or to elicit some other response desired by the advertiser. Advertisement is distinguished from other forms of communication in that the advertiser pays the media to deliver the message."(Encyclopedia Britannica: 1994,Vol I). I prefer the definition put forwarded by William F. Arenas and Courtland L. Bovee, which is a quite comprehensive one: "Advertisement is the no personal communication of information, usually paid for and usually persuasive in nature, about products (goods and services) of ideas by identified sponsors through various media."(Bovee: 1994, 68) 
Advertisement is a gigantic system. It can be classified into different types according to different criteria. Broadly speaking, there are three main types of advertisement: commercial advertisement, social advertisement and public advertisement. The contents discussed in the following part will mainly talk about the characteristics of commercial advertisement. The commercial advertisement always leads to the behavior of purchasing the products or consuming the recommended service. Commercial ads are much more presented through mass media for the reason that manufacturers and companies are willing to spend a large sum of money to make a certain product known or to boost the image of a brand. Commercial advertising can also be divided into two groups according to the target audience. They are consumer advertising and business advertising. Most of the ads in the mass media are consumer advertising, so this paper will concentrate on the characteristic of it.

Mental Lexicon. Mental lexicon, put forward in the last century, is a new research subject in Linguistics, which is a critical step in making language acquisition research shift from a linguistic perspective to a new perspective: linguistic \& psychological perspective. Treisman, A.M. who is the first to propose the concept of mental lexicon in 1964, holds that the mental lexicon is a repository of all the information that a reader or a listener has concerning words of a language. According to him, it is in every speaker's mind that exists a well organized system of lexical representation which consists of word's spelling, sound and meaning. Different definition of this term has been given by different scholars. The definition of mental lexicon given by J.C. Richards et al. (2000) is a mental system that contains all the information a person knows about words.

Spreading Activation Models. A alternative model was propose by modifying of the rigid hierarchical assumptions while retaining the concept of network. This kind of models is referred to as spreading activation models that first proposed by Collins and Loftus(1975). The model suggests that mental lexicon is not stored in a strict hierarchical order in the network. In contrast, the organization is a network of interconnected elements and the distance between the nodes is determined by functional factors and structural property. Thus the retrieval of a word becomes a spread of activation.

Hierarchical Network Models. The hierarchical model, which was the most earliest and the most influential model of L1 mental lexicon, was proposed by Collins and Quillians in 1969. In a hierarchical network, the network is hierarchical with some elements standing above or below others. It resembles the semantic field theory in which there are super ordinates, subordinates and coordinates.

Word extraction. Netherlands linguists Forster (1976) regarded mental lexicon as a library. A word is like a book, which is placed on the mental lexicon / in the Library, its location can be found from the index directory. He believes that these card catalogues are called extract file (access files), they are orthographic access file for recognizing the written words, phonological access file for identification of spoken language to extract file semantic / syntactic file for identification the semantics the lexical extracted files, with three extracted files, lexical items can be read and understood, search out in listening and speaking.

\section{The Application of Mental Lexicon in Advertising English Vocabulary}

The Application of Spreading Activation Models. In the following examples, we could see how this model is implied. At first, I will take the ad words of apple computer for example. "Apple thinks different." As we all know, the term "apple" is a kind of fruit, so it is easy to stimulate readers' mental dictionary about different fruits, such as: cherry; pear; peach; etc. at the same time. The color of ripe apple is red, no doubt, the other color terms will come to person's mind, such as: orange; green; blue; etc. besides this, red is the color of rose, which stands for the strong emotion between the two lovers. With the further mental dictionary, different flowers will be listed, for instance: violet; lotus; narcissus; etc. When we come to the word "different", with the guiding of this model, the following words will be put forward. Such as: style; color; fashion; size; character; speed ; and so on, because there are so many differences in our daily lives. 
The Application of Hierarchical Network Models. The research of Collins and Quillian(1969) is a prototype of this approach. In the model, concepts similar to the word are represented as distinct nodes in a network of category relations that deal with hyponym and property relations that indicate what characteristics may be attributed to the items at various levels in the network. The model is shown in the following example: The choice of a new generation. (Pepsi). I will pick the word "generation" for example. Generation---humankind; animal. And then I will use the following figure to demonstrate the word "animal" in hierarchical network models.

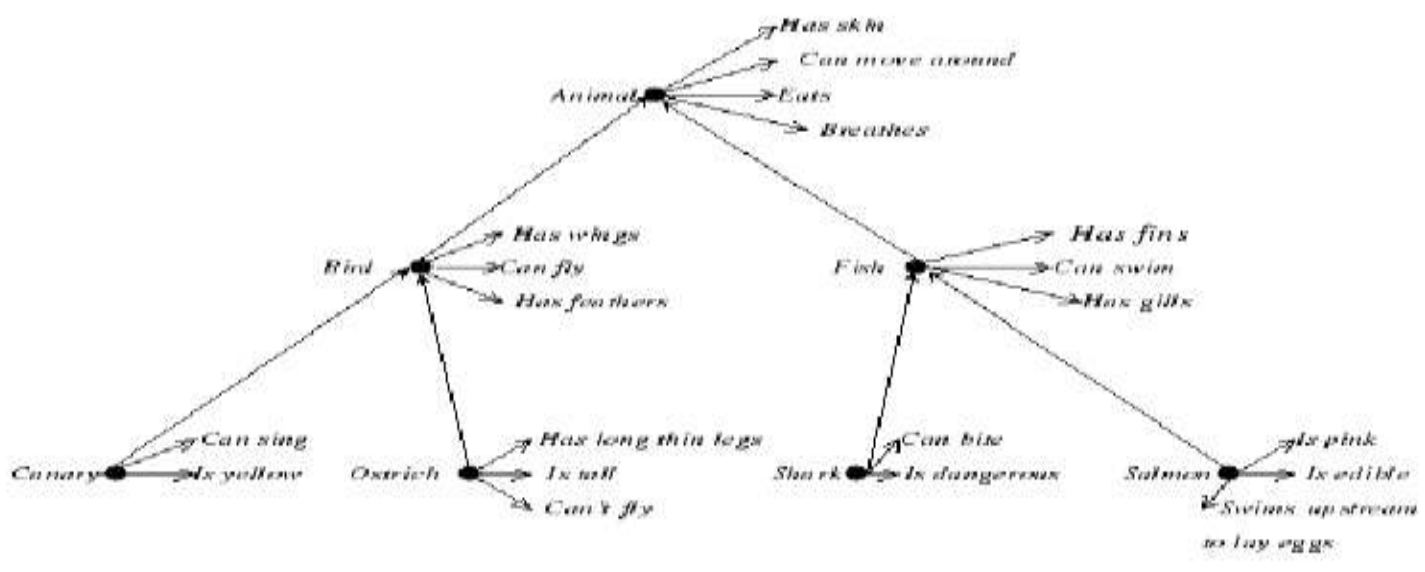

Figure 1.

\section{Factors Affecting Lexical Extraction and Organization in Ad}

When reading a word, another word which is related to the word will come to the reader's mind, but the contact or the relation between them is not exactly the same. the researchers have found that the organization of the mental lexicon follows two major principles: the meaning and syntax. Lexical extraction and organization usually are affected by the use of the word frequency, word structure, context and semantic factors.

The High Frequency of the Word. Psychologists have found that frequency plays an important role in the lexical model. Generally speaking, the higher the frequency of the use of the word is, the more likely it is to be extracted. Adjective words have rich emotional and rendering power, and its main function is to describe and modify a noun, making the pale entities suddenly become vivid and natural, fascinating. For example: 1) The taste is great. (Nestlé) ; 2) Good teeth, good health. (Colgate toothpaste), from the above examples, we could easily find adjectives are a high frequency word in people's mind used to modify the quality and function of products.

Contextual Factors. Each language has a lot of polysemy, context can contribute to the identification of the word, to resolve the ambiguity of the word. Many experiments proved context has a great impact on the mental lexicon, the isolated cognitive words through context are easily to be understood than the word itself being isolated. For example: Eye drops ads: please rotate you eyes several times, letting the syrup cover the whole world. From this context, it is natural for the readers to think of the phrase "the whole world" is said to the whole eyeball, instead of the world.

Semantic Factors. Semantic relationships between words are the main contact. Recognizing the word with semantic links is faster than words without semantic links. I will take shampoo ad for instance. Start Ahead(Rejoice) : this is ad of Rejoice shampoo which is a famous brand. Comparatively speaking, on hearing this ad words, the word come to people's mind must be "hair" which has close semantic link with the word "head" rather than the word "shampoo" without semantic link.

Lexical Effect. Lexical effect is also known as the true word or non-word effect, it refers to some of the words have been set up as a glossary entries, when words are identified, vocabulary entries are 
considered superior to the correspondence relationship voice or letter .Legal non-words accord to word formation and spelling rules of the English, but there is no word. Such as: An ad of a Holiday Inn uses the word "Twogether" as it's ad title . They deliberately write "Twogether" to replace "Together", No doubt, it influences the speed of understanding to this ad, but it actually improves advertising rendering by changing the words and shows a novel conception that the men and women (two persons) share the happy and romantic holiday together .

\section{Conclusion}

In summary, the advertising English shows different characteristics with other stylistic at all levels. Vocabulary in advertising English has its own unique characteristic, Advertising English develops fast with the development of society. The designers of ad, with knowing the factors of person's vocabulary acquisition, they could design and produce more and more ad with fresh feeling and new quality and achieve business profit. For consumers or readers, understanding the intention of words use by designers not only enables them to further understand of the charm of the English language, but also provides them with another perspective to observe the concept of social life and the values of the modern English-speaking countries.

\section{Reference}

[1] $\mathrm{Hu}$ Yueyue, Cheng Jia Cai, Wu Xiaofang. The lexical features of advertising English [J]. Changchun Normal University Journal, 2016 (07)

[2] Bauman. From the perspective of advertising psychology, the characteristics of advertising English vocabulary [J]. overseas English, 2014 (10).

[3] Xing Shuping. Analysis of the characteristics of advertising English language [J]. Journal of Jincheng Institute of Technology, 2012 (05).

[4] Wang Haibo, Du Haifang. Analysis of the characteristics of advertising English language [J]. China business, 2011 (06).

[5] Classic English advertising language

[6] Dai Xinxin. The language features of classic advertising English under the background of consumerism [J]. news enthusiasts, 2017 (11).

[7] Liu Qianyu, Li Haihong. The linguistic features of advertising English from a linguistic perspective, [J]., 2017 (06).

[8] Lexical representation and development in a second language[J] . N Jiang. Applied Linguistics . 2000 